\title{
Spontaneous occlusion of a cerebral arteriovenous malformation after subtotal endovascular embolisation
}

\author{
A. Chiriac, N. Dobrin ${ }^{\star}$, A.St. Iencean, I. Poeata \\ "Grigore T. Popa" University of Medicine and Pharmacy, Iasi \\ "Prof. Dr. N. Oblu” Clinic Emergency Hospital, Iasi*
}

\begin{abstract}
We present a case of a patient with a ruptured temporo-occipital arteriovenous malformation, grade 3 on Spetzler-Martin scale, with an unpredictable evolution. A spontaneous occlusion of the malformation was found at 4 months after the initial partial endovascular embolization. We reviewed and discuss some aspects concerning clinical and angiographic finding, the angioarchitectural remodelling and evolution of the AVMs after partial occlusion treatment.
\end{abstract}

\section{Introduction}

It is known that, even at present, the natural history of arteriovenous malformations of the brain is not fully clarified. Another highly discussed is the evolution of these lesions after their partial occlusion and especially the one related to their potential for spontaneous regression.

Regression of cerebral arteriovenous malformations either spontaneous or following bleeding, partial surgery or embolization is a well-known occurrence. This phenomenon was reported in 2-3\% of all partially treated lesions. The goal of the following case report was to document a case of cerebral arteriovenous malformations spontaneous disappearance after partial, uncomplicated endovascular treatment focusing on its angioarchitectural remodelling over time $[1,2]$.

\section{Case presentation}

A 48-year-old man was admitted to hospital for a secondarily generalized seizure. His family and medical history were unremarkable. On admission, the patient was in a postictal coma and neurological examination did not reveal any focal neurological deficits. The patient underwent a cerebral computed tomography (CT) examination that showed an occipital area of low attenuation suggesting for an arteriovenouse malformation. Also, a magnetic resonance (MR) examination of the brain, performed on a superconducting $0.5 \mathrm{~T}$ system, showed an arteriovenous malformation (AVM) in the left occipital lobe. No mass effect or acute / chronic bleeding was noted. The EEG examination didn't reveal any electrical abnormalities. 


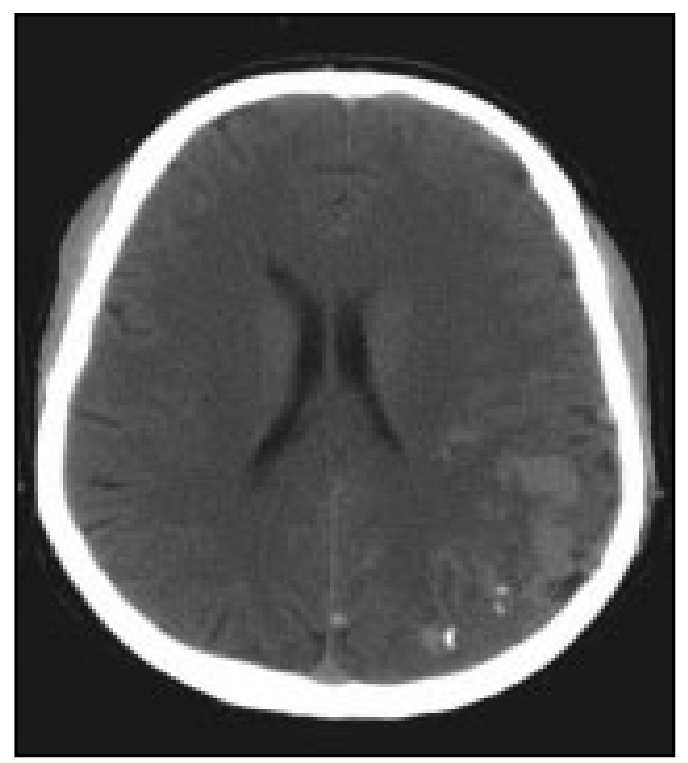

Figure 1 - Native CT suggesting for an arteriovenouse malformation

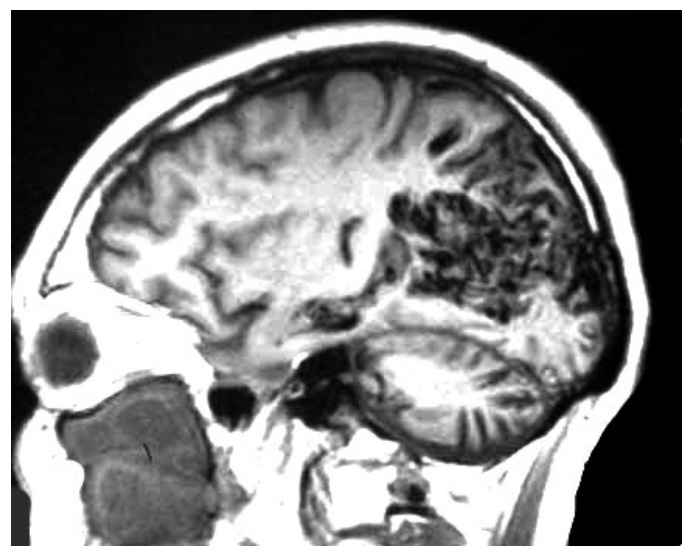

Figure 2 - T2 sequence suggesting for an arteriovenouse malformation

Digital subtraction angiography (DSA) of intracranial vessels revealed an occipital AVM with a $3 / 3 / 3 \mathrm{~cm}$ trapezoidal nidus. The lesion was supplied from both anterior and posterior circulation. From anterior circulation we identified two major arterial pedicles originating from distal M2 segment of left middle cerebral artery. The supply from posterior circulation is made via two arterial pedicles from P2 segments of the left posterior cerebral artery. Venous drainage occurred by means of two veins coursing to the superior sagittal sinus and one deep vein. The AVM was classified as a grade 3 according to SpetzlerMartin grading system. Endovascular treatment of the AVM was decided on the basis of the patient's symptoms and location.

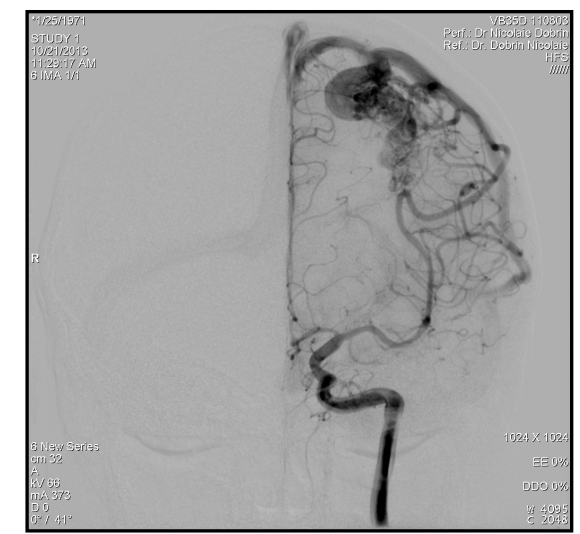

Figure 3 - DSA left ICA - face view showing an occipital AVM

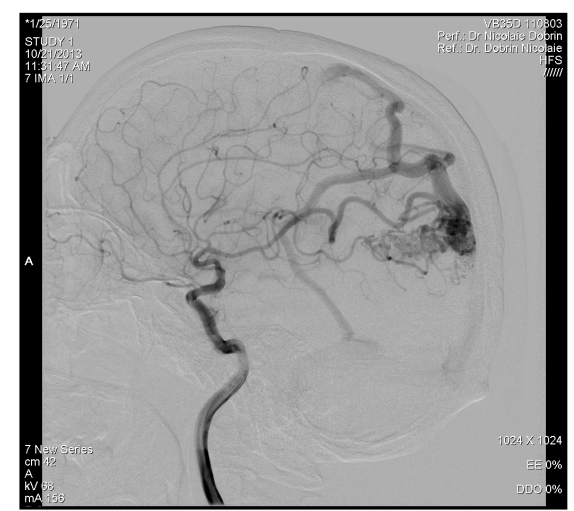

Figure 4 - DSA left ICA - lateral view showing an occipital AVM 


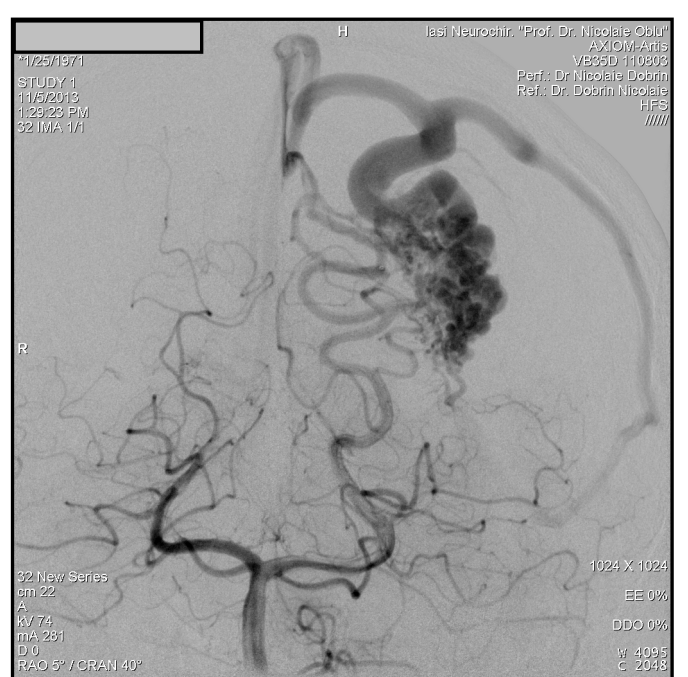

Figure 5 - DSA left Vert. A - face view showing an occipital AVM

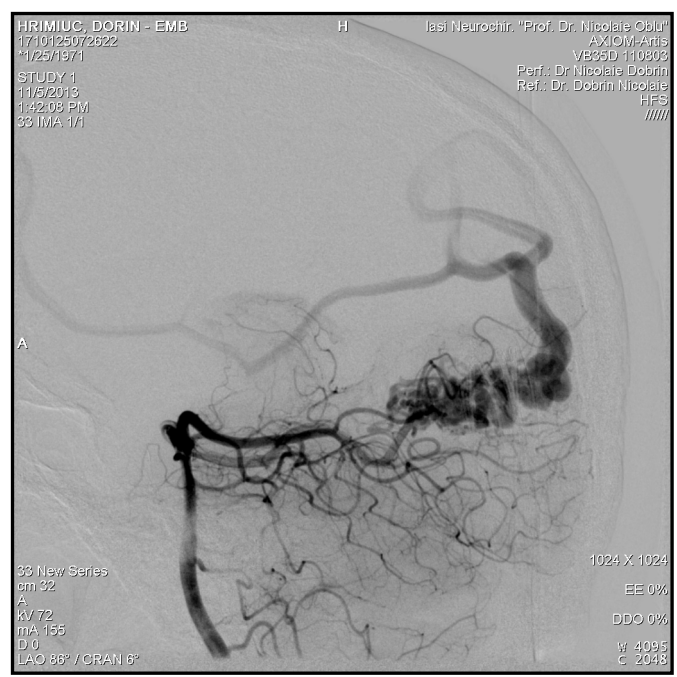

Figure 6 - DSA left Vert. A - lateral view showing an occipital AVM

Four consecutive arterial pedicle injections of 0.4 and $0.2 \mathrm{cc}$ mixture of a polymerizing agent (Histoacryl, Braun) and Lipiodol UP was performed.

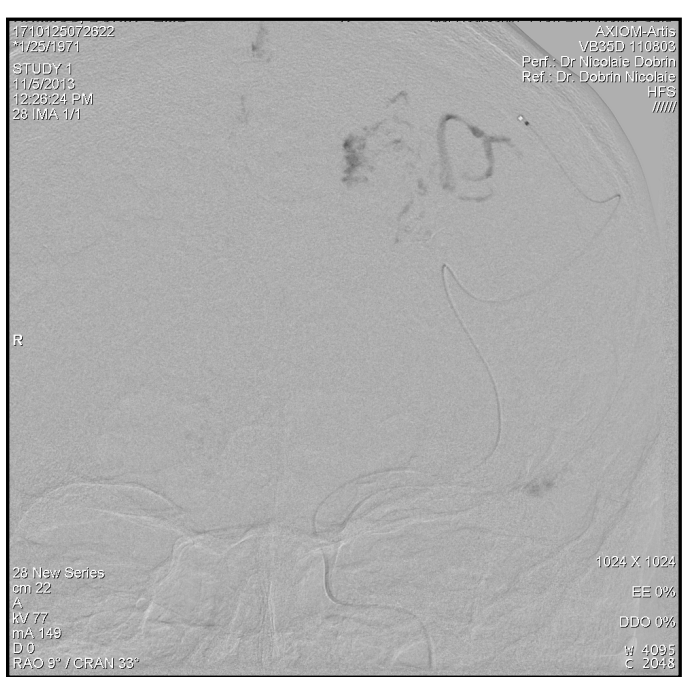

Figure 7 - DSA - Glue injection on arterial pedicles originating from distal M2 segment of left middle cerebral artery

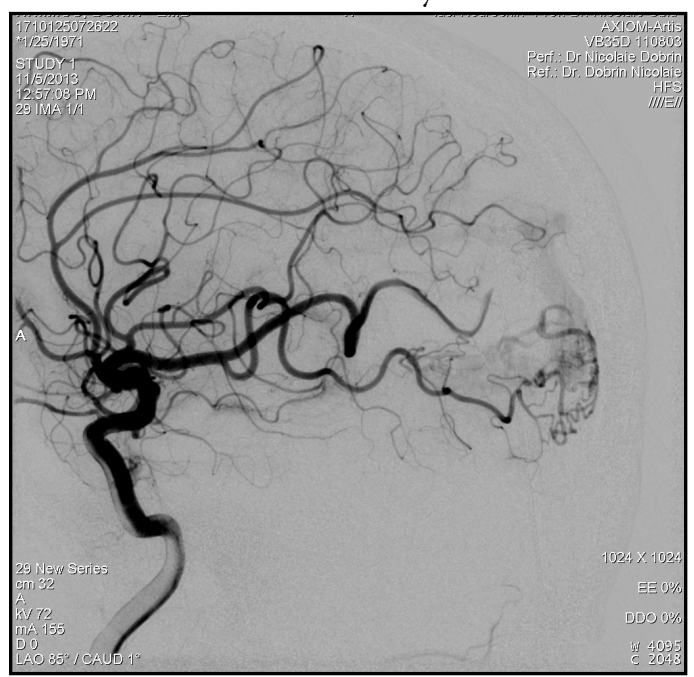

Figure 8 - DSA left ICA - lateral view showing a subtotal devascularization of AVM

The final angiographic control showed a subtotal devascularization of the AVM with a remnant of supply through a small artery from distal left Sylvian artery and one from left posterior cerebral artery. 
The post-embolisation CT control revealed the glue cast in the AVM with absence of ischemic or haemorrhagic signs. The clinical course was uneventful and the patient was discharged 7 days later.

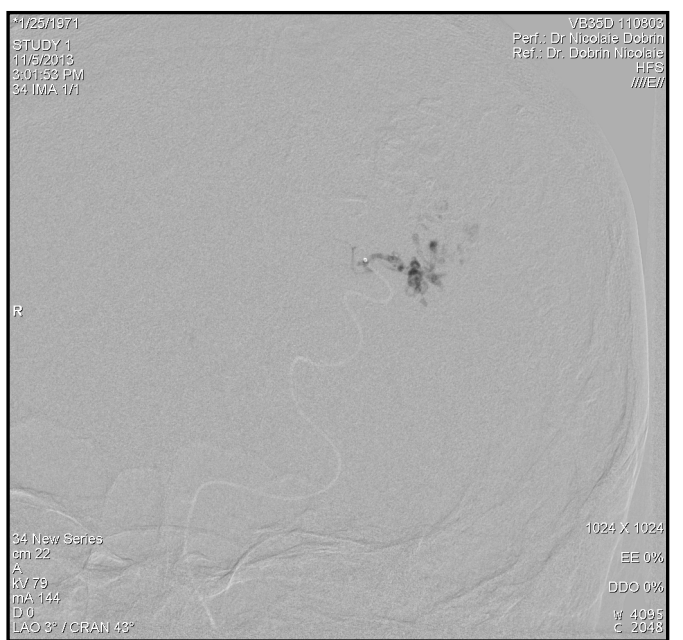

Figure 9 - DSA - Glue injection on arterial pedicles originating from $\mathrm{P} 2$ segments of the left posterior cerebral artery

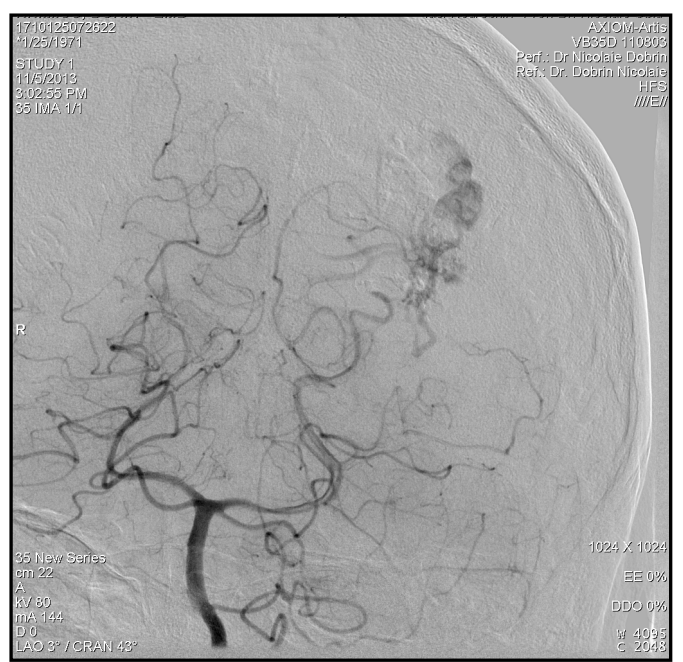

Figure 10 - DSA left Vert. A - face view showing a subtotal devascularization of AVM
Four months later a follow-up cerebral DSA examination showed a complete disappearance of abnormal vessels with no filling of the AVM. Evaluation of the capillary phase showed no early draining vein apperance.

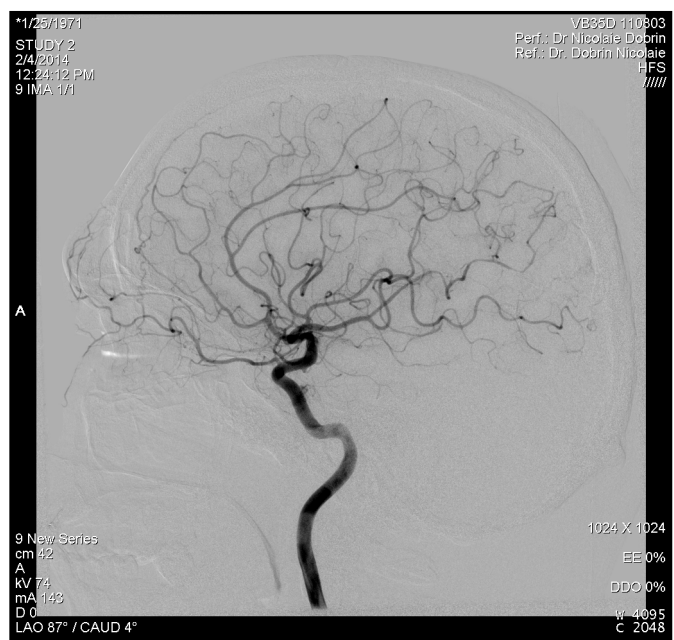

Figure 11 - DSA Control at 4 month - left ICA lateral view showing a total occlusion of AVM

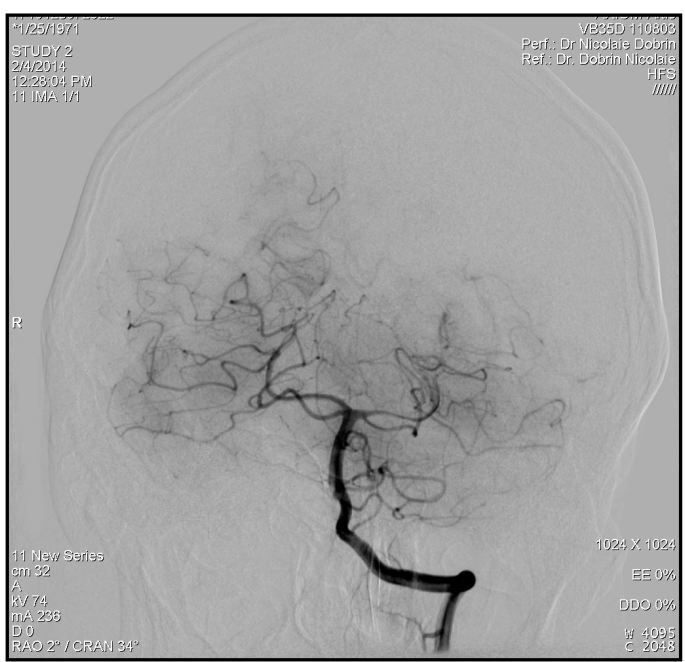

Figure 12 - DSA Control at 4 month - left Vert. A. face view showing a total occlusion of AVM 
DOI: 10.2478/romneu-2014-0024

\section{Discussions}

Cerebral arteriovenous malformations are considered dynamic vascular lesions.

Their angioarchitectural feature concerning arterial supply and/or venous drainage may suffer spontaneous changes. Thus, they can increase in size or even to evolve to complete spontaneously thrombosis. Spontaneous regression or obliteration of cerebral arteriovenouse malformation is a very poorly understood a phenomenon that was infrequently reported. Their predisposing factors are still discussed.

Spontaneous obliteration of cerebral AVMs is a rare event that was reported to occur in $0.8 \%$ to $2 \%$ of cases. Although, spontaneous obliteration does rarely occur with larger AVMs, being more common in small ones. Stated reasons for spontaneous obliteration include compressive haemorrhage due to AVM rupture, premature atherosclerosis, hemodynamic changes associated with treatment (embolization or surgery) embolus, turbulence in feeding vessels, elevated estrogens, kinking of feeding vessels as a result of gliosis [1,2].

Since most of the literature dates did not reported a preponderance of females in their cohort, it is difficult to support the theory that estrogens play a real role in spontaneous AVMs obliteration.

It was certainly demonstrated that partial embolization even surgery for hematoma evacuation or for intended resection may remove enough nidus for an alteration in flow pattern to take place and lead to a subsequent spontaneous occlusion [1,2].

Patel and all showed that the mass effect due to parenchymal haemorrhage may be a contributory factor of spontaneous AVM occlusion, possibly as a consequence of stretching and narrowing of the feeding arteries, occlusion of the draining veins, or obliteration of the nidus [1,2].

Other authors have noted that dimension, vascular configuration and location of the cerebral AVM may be a predictive factor for a spontaneous obliteration evolution. Thus, it is more likely to have a spontaneous obliteration if the AVM is small, superficial, fed by a single artery, or drained by a single vein.

In their individual study Patel and coauthors have showed that progressive venous restriction leading to venous occlusion in the presence of a protective factor may offer an explanation for spontaneous AVM obliteration. The protective factors may include, for example, a paucity of feeding vessels, or long feeding vessels with a large pressure drop along their length, or spasm of feeding vessels in the presence of hemorrhage. The presence of these factors may prevent an increase in intranidal pressure during venous occlusion, thereby limiting the size of hemorrhage from the nidus. Large variations in feeding artery pressure have already been reported, with smaller AVMs having higher feeding artery pressure than larger ones and those that present with hemorrhage having higher feeding artery pressure than those with nonhemorrhagic presentations $[1,2]$.

The tortuosity and altered haemodynamic properties of feeding arteries have been also demonstrated another possible factors 
triggering AVM occlusion. They can contribute to the formation of intranidal thrombi because of stasis, eddy currents and turbulent flow.

A retrograde propagation of a venous thrombus may lead to the spontaneous occlusion of the entire AVM. This situation may occur in the presence of a venous outlet obstruction heralded by a post-stenotic dilatation of the draining veins.

\section{Conclusions}

Spontaneous regression of cerebral arteriovenouse malformations cannot be clinical or angiographical predicted. Moreover, it should not be considered as an option in their therapeutically management, despite its proven occurrence.

\section{Correspondence}

A.St. Iencean

andrei_steffan@yahoo.com

\section{References}

1. Maneesh C. Patel, Timothy J. Hodgsona, Andras A.
Kemenya and David M. Forstera: Spontaneous Obliteration of Pial Arteriovenous Malformations: A Review of 27 Cases, AJNR 2001 22: 531-536;

2. Mangiafico S., Cellerini M., Villa G., Nistri M., Ammannati F., Giordano G.P.: Disappearance of a Cerebral Arteriovenous Malformation after Partial Endovascular Embolisation, Interventional Neuroradiology 2001, 7: 41-46;

3. Cellerini, M., Mangiafico, S., Villa, G., Ammannati, F., \& Giordano, G. P. (2003). Disappearance of cerebral arteriovenous malformations after partial endovascular embolisation: four cases with follow-up. Neuroradiology, 45(12), 916-920.

4. Alejandro Torrico, T., \& José Tevah, C. (2006). Spontaneous occlusion of previously cerebral embolized residual arteriovenous malformation: report of two cases. Arquivos de neuro-psiquiatria, 64(3A), 681-685.

5. Clarençon, F., Biondi, A., Sourour, N. A., Di Maria, F., Iosif, C., Nouet, A., ... \& Chiras, J. (2013). Spontaneous closure of intracranial dural arteriovenous fistulas: A report of 3 cases. Clinical neurology and neurosurgery, 115(7), 971-975.

6. Abdulrauf SI, Malik GM, Awad IA.(1999) Spontaneous angiographic obliteration of cerebral arteriovenous malformations, Neurosurgery. Feb;44 (2):280-7; discussion 287-8.

7. Buis DR, van den Berg R, Lycklama G, van der Worp HB, Dirven CM, Vandertop WP. (2004) Spontaneous regression of brain arteriovenous malformations--a clinical study and a systematic review of the literature, J Neurol. Nov;251(11):1375-82. 